\title{
Antioxidant properties of proanthocyanidins of Uncaria tomentosa bark decoction: a mechanism for anti-inflammatory activity
}

\author{
Cristina Gonçalves ${ }^{a, b}$, Teresa Dinis ${ }^{b, *}$, Maria Teresa Batista ${ }^{a}$ \\ a Laboratorio de Farmacognosia, Faculdade de Farmácia and Centro de Estudos Farmacêuticos, \\ Universidade de Coimbra, 3000-295 Coimbra, Portugal \\ ${ }^{\mathrm{b}}$ Laboratorio de Bioquímica, Faculdade de Farmácia and Centro de Neurociências e Biologia Celular de Coimbra, \\ Universidade de Coimbra, r/c, 3000-295 Coimbra, Portugal
}

Received 16 April 2004; received in revised form 21 October 2004

Available online 9 December 2004

\begin{abstract}
Decoctions prepared from the bark of Uncaria tomentosa (cat's claw) are widely used in the traditional Peruvian medicine for the treatment of several diseases, in particular as a potent anti-inflammatory agent. Therefore, the main purpose of this study was to determine if the well-known anti-inflammatory activity of cat's claw decoction was related with its reactivity with the oxidant species generated in the inflammatory process and to establish a relationship between such antioxidant ability and its phenolic composition.

We observed that the decoction prepared according to the traditional Peruvian medicine presented a potent radical scavenger activity, as suggested by its high capacity to reduce the free radical diphenylpicrylhydrazyl, and by its reaction with superoxide anion, peroxyl and hydroxyl radicals as well as with the oxidant species, hydrogen peroxide and hypochlorous acid. It also protected membrane lipids against peroxidation induced by the iron/ascorbate system, as evaluated by the formation of thiobarbituric acidreactive substances (TBARs). The decoction phenolic profile was established by chromatographic analysis (HPLC/DAD and TLC) revealing essentially the presence of proanthocyanidins (oligomeric procyanidins) and phenolic acids, mainly caffeic acid.

Thus, our results provide evidence for an antioxidant mechanism underlying the anti-inflammatory activity of cat's claw and support some of the biological effects of proanthocyanidins, more exactly its antioxidant and radical scavenging activities.
\end{abstract}

(C) 2004 Elsevier Ltd. All rights reserved.

Keywords: Uncaria tomentosa; Decoction; Proanthocyanidins; Antioxidant; Scavenger; Anti-inflammatory activity

\section{Introduction}

Uncaria tomentosa (Willd.) DC., Rubiaceae, grows in the Amazon forests of Peru, where it is known as "uña de gato" (cat's claw). Decoctions prepared from the bark have been widely used in traditional Peruvian medicine for the treatment of several diseases, in particular as potent anti-inflammatory agents (Desmarchelier

\footnotetext{
${ }^{*}$ Corresponding author. Fax: +351239852569.

E-mail address: tcpdinis@ci.uc.pt (T. Dinis).
}

et al., 1997; Sandoval et al., 2000). Although previous studies have supported some of their pharmacological properties, namely the antimutagenic (Rizzi et al., 1993; Sheng et al., 1998) and the anti-inflammatory actions (Aquino et al., 1991; Piscoya et al., 2001), the molecular mechanisms underlying such activities have not yet been clarified. The capacity of these decoctions to inhibit either the TNF $\alpha$ production, or to a lesser extent the $\mathrm{PGE}_{2}$ production, may explain some of their anti-inflammatory properties (Sandoval et al., 2000; Piscoya et al., 2001). Additionally, it has been reported that they possess antioxidant activity, as evaluated by the 
reaction with the free radical 1,1-diphenyl-2-picrylhydrazyl (DPPH) (Sandoval et al., 2000). Nevertheless, we are aware that a better characterization of this antioxidant capacity requires model systems and methodologies involving the generation of diverse reactive species, namely superoxide anion $\left(\mathrm{O}_{2}^{--}\right)$, peroxyl (ROO'), and hydroxyl (HO') radicals, hydrogen peroxide $\left(\mathrm{H}_{2} \mathrm{O}_{2}\right)$, and hypochlorous acid $(\mathrm{HOCl})$, as well as the evaluation of the potential ability to decrease them (Halliwell, 1997). Although the production of such reactive species occurs during the normal metabolism in all the aerobic organisms, it may be dramatically increased in the inflammatory processes.

It is well known that the central feature of inflammatory activity is the activation of phagocytic cells that synthesize and release large amounts of reactive species causing cell and tissue injury, either directly by oxidative degradation of essential cellular components or indirectly by altering the normal protease/antiprotease balance (Halliwell et al., 1988). Providing that reactive oxygen species may act as toxins, mediators and modulators of inflammatory gene activation, efforts have been directed to investigate antioxidant molecules as potential therapeutic agents (Conner and Grisham, 1996). In this context, polyphenols becoming increasingly important (Korkina and Afanas'ev, 1997; Groot and Rauen, 1998; Packer et al., 1999). Epidemiological studies have suggested a correlation between the consumption of polyphenol-rich foods or beverages and the prevention of diseases (Maxwell, 1995; Arnao et al., 2001). Indeed, polyphenols are reducing agents and like vitamin $\mathrm{C}$ and vitamin $\mathrm{E}$ they possess potential for protection against oxidative damage (Rice-Evans et al., 1996; Bagchi et al., 2000).

On the other hand, concerning the Uncaria chemical constituents, previous studies have been essentially focused on its alkaloid components (Laus and Keplinger, 1994; Mohamed et al., 2000; Muhammad et al., 2001). Thus, the aim of this study was to evaluate the polyphenolic composition and the scavenging properties of an $U$. tomentosa decoction, prepared according to traditional Peruvian medicine and to establish a relationship between phenolic composition and antioxidant activity.

The assessment of its general free radical scavenging ability was got by the interaction with DPPH in solution. To better characterize this antioxidant potential, the interactions of the decoction with the superoxide, hydroxyl and peroxyl radicals as well with hydrogen peroxide and hypochlorous acid generated in homogeneous model systems were evaluated. Also, the effect on lipid peroxidation, initiated by $\mathrm{Fe}^{2+} /$ ascorbate in sarcoplasmic reticulum (SR) membranes, used as a native membrane model, was assessed by TBARs formation. Attempting to establish a relationship between its polyphenolic constituents and antioxidant capacity, a decoction depleted of tannins was prepared and its reacting efficiency with DPPH determined. Furthermore, the decoction was fractionated by gel chromatography and analyzed by HPLC and TLC. The proanthocyanidin (condensed tannins) content was also determined.

\section{Results and discussion}

\subsection{Free radical scavenger activity}

The general free radical scavenger activity of the decoction was evaluated by its interaction with DPPH in solution. DPPH is a stable free radical that can accept an electron or hydrogen radical to become a stable diamagnetic molecule. Because of its odd electron, the methanolic solution shows a strong absorption band at $517 \mathrm{~nm}$ (Blois, 1958), which decreases in the presence of free radical scavengers. DPPH scavenging activity has been largely used as a quick and reliable parameter to assess the in vitro general antioxidant activity of plant extracts (Soares et al., 1997; Bonina et al., 2000; Munasinghe et al., 2001), that has been assigned to phenolic compounds namely, phenolic acids and flavonoids (Rice-Evans et al., 1996; Van Acker et al., 1996).

Uncaria tomentosa decoction promptly reacted with DPPH leading to a loss of $70 \%$ in absorbance intensity. In contrast, the tannin-free decoction only presented $7 \%$ of DPPH reduction capacity (Fig. 1). A correlation between the capacity of DPPH reduction and the proanthocyanidins content (grey bar in the center) was also verified. These results clearly indicate that the loss of proanthocyanidins leads to a significant loss of antioxidant capacity.

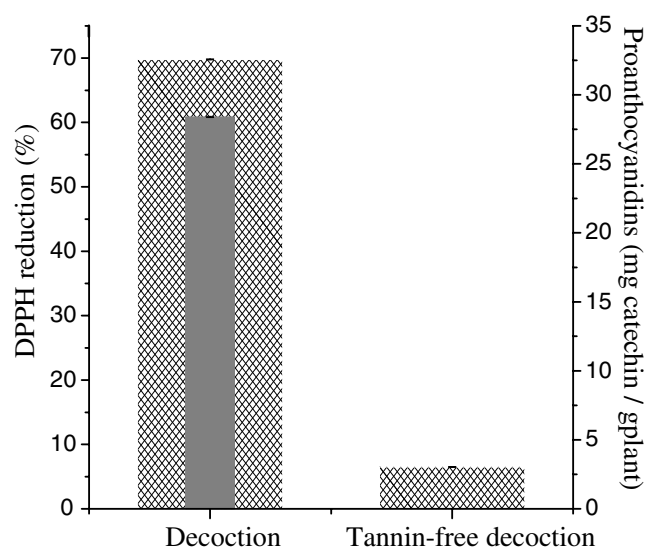

Fig. 1. Relationship between DPPH radical quenching of decoction and tannin-free decoction in terms of percentage of DPPH reduction and proanthocyanidins content, as expressed by $\mathrm{mg}$ of catechin/g plant. The great columns (crossed lines) represent the capacity of DPPH reduction of both decoction and tannin-free decoction. Proanthocyanidins were only detected in the decoction as evidenced by the grey column in the center. Bars represent the mean \pm SD of at least three different experiments. 
As already referred, the aim of this study was mainly to identify the major constituents of $U$. tomentosa decoction responsible for its antioxidant activity and more specifically for the scavenging capacity of reactive species involved into the inflammatory process.

\subsection{Chemical characterization}

An HPLC profile of the $U$. tomentosa decoction recorded at 280 and $320 \mathrm{~nm}$ is illustrated in Fig. 2. The phenolic profile was established according to the retention characteristics in reversed-phase and/or UV spectra, using HPLC/DAD analyses before and after fractionation on Sephadex LH-20 and tannins elimination (Table 1). The identification of the compounds present in the decoction was tentatively made on the basis of their retention and/or spectral characteristics in comparison with authentic samples.

The confirmation of the presence of protocatechuic acid, catechin, epicatechin, vanillic acid, caffeic acid and syringic acid, was assessed by the addition of authentic samples to the decoction or to the tanninfree decoction. In these cases, no deviations were found neither in the retention times nor in the UV spectra.

HPLC/DAD analyses were consistent with the major presence of flavan-3-ol related compounds (proanthocyanidins). A few ellagic derivatives were found but gallates were not detected. Phenolic acids and their derivatives were also found, being caffeic acid the most representative. Yet, some phenolic compounds could not be well identified because its co-elution with others. In order to establish a better characterization of the decoction constituents, fractionation through a Sephadex LH-20 column was carried out. Nine fractions were separated according to UV behaviour, but only three of them $\left(\mathrm{F}_{\mathrm{I}}, \mathrm{F}_{\mathrm{II}}\right.$, and $\left.\mathrm{F}_{\mathrm{III}}\right)$ were chemically distinct. As represented in Table 1, the fraction I contained caffeic acid, as the main constituent, protocatechuic acid and catechin, as well as some constituents with spectra suggesting flavonols (quercetin and kaempferol) and phenolic acid derivatives (ellagic and $p$-coumaric). In addition, epicatechin and flavan-3-ol related compounds were detected in small quantities. Higher concentrations of these compounds occurred in fraction II. Fraction III also showed peaks corresponding to flavan-3-ol related compounds. Vanillic and syringic acids could only be identified in the tannin-free decoction, since they were present in low concentrations and co-eluted with the proanthocyanidins.

Complete acid hydrolysis of both fractions II and III led to the disappearance of flavan-3-ol derivatives and to the simultaneous appearance of cyanidin, showing the presence of proanthocyanidins, mainly of the procyanidin type. Concerning the polymerisation degree of proanthocyanidins, TLC analysis revealed that fraction II was constituted by oligomeric procyanidins, essentially dimers and trimers whereas more polymerised proanthocyanidins (oligomers greater than four flavan-3-ol subunits) were detected in fraction III.

The results obtained illustrate that the majority of the detected phenolic compounds are proanthocyanidinlike, suggesting that the radical scavenging effectiveness of $U$. tomentosa decoction can be related to them. Proanthocyanidins have been reported to provide protection against free radicals and oxidative stress, possessing important biological, pharmacological and therapeutic properties (Packer et al., 1999; Bagchi et al., 2000; Santos-Buelga and Scalbert, 2000), namely anti-inflammatory activity (Li et al., 2001).

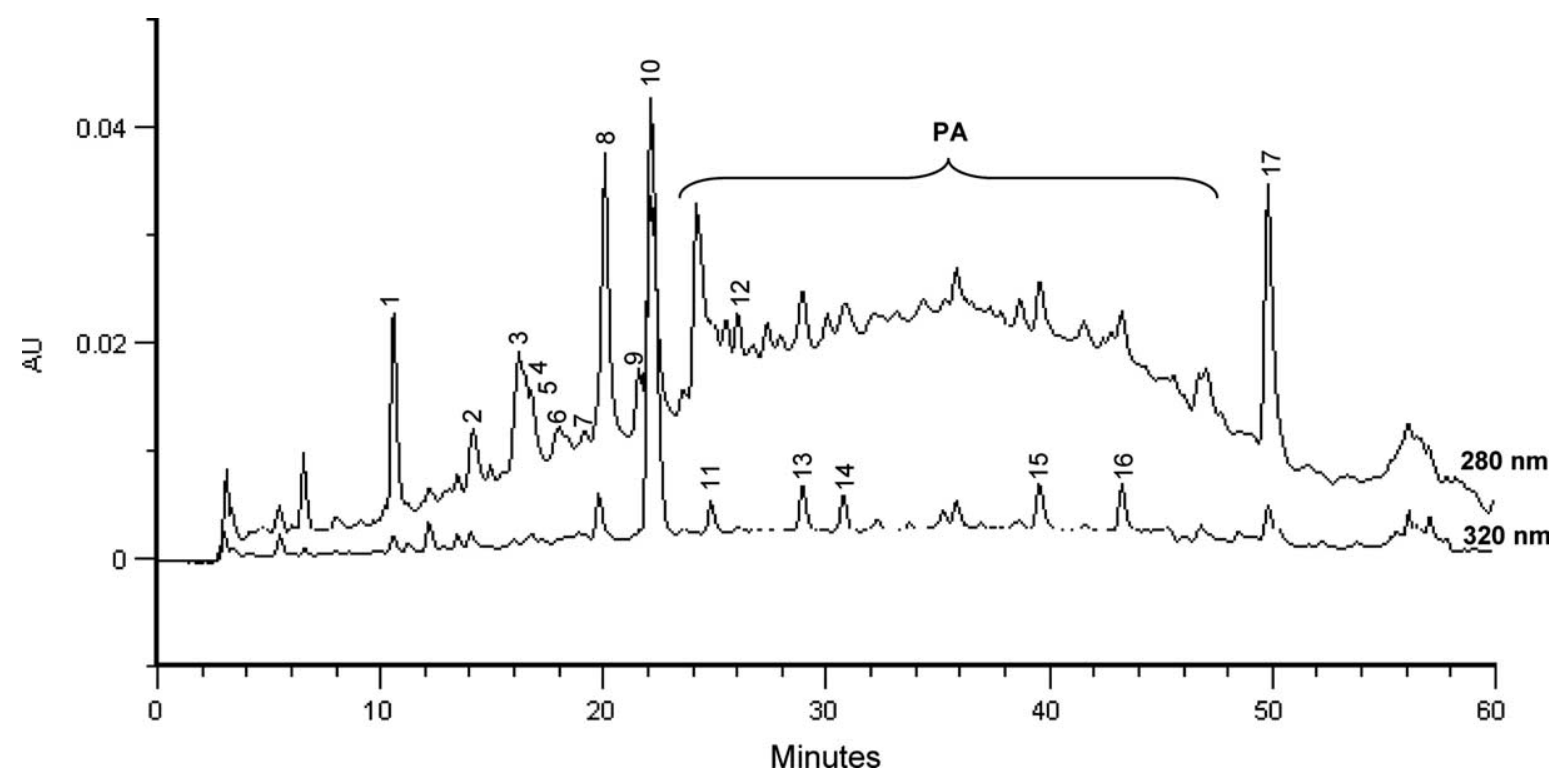

Fig. 2. A typical HPLC profile of Uncaria tomentosa decoction recorded at 280 and $320 \mathrm{~nm}$. Peaks identification is shown in Table 1. 
Table 1

HPLC phenolic composition of the Uncaria tomentosa decoction

\begin{tabular}{|c|c|c|c|c|c|c|c|}
\hline Peak number & $R_{\mathrm{t}}(\mathrm{min})$ & $\lambda_{\max }(\mathrm{nm})$ & Compound $^{\mathrm{a}}$ & $F_{I}$ & $F_{I I}$ & $\mathrm{~F}_{\text {III }}$ & Tannin-free decoction \\
\hline 1 & 10.6 & $259 ; 293 \mathrm{sh}$ & Protocatechuic acid & + & & & + \\
\hline 2 & 14.1 & 279 & Proanthocyanidin & & + & & \\
\hline 3 & 16.2 & 279 & Proanthocyanidin & & + & & \\
\hline 4 & 16.4 & 279 & Catechin & + & & & \\
\hline 5 & 16.8 & 279 & Proanthocyanidin & & + & & \\
\hline 6 & 18.0 & 279 & Proanthocyanidin & & + & & \\
\hline 7 & 19.2 & 279 & Proanthocyanidin & & + & & \\
\hline 8 & 20.1 & 279 & Epicatechin & & + & & \\
\hline 8 & 20.1 & 279 & Proanthocyanidins & & + & + & \\
\hline 9 & 21.6 & $261 ; 292$ & Vanillic acid & & & & + \\
\hline 9 & 21.6 & 279 & Proanthocyanidins & & + & + & \\
\hline 10 & 22.1 & $298 ; 323$ & Caffeic acid & + & & & + \\
\hline 11 & 24.8 & $280 ; 336 \mathrm{sh}$ & Acylated flavan-3-ol derivative & & & & \\
\hline 12 & 26.0 & 275 & Syringic acid & & & & + \\
\hline 13 & 28.9 & $282 ; 309 \mathrm{sh}$ & $p$-Coumaric acid derivative & & & & + \\
\hline 14 & 30.8 & $281 ; 309 \mathrm{sh}$ & $p$-Coumaric acid derivative & + & & & + \\
\hline 15 & 39.6 & $258 ; 266 \mathrm{sh} ; 285 \mathrm{sh} ; 354$ & Quercetin derivative & + & & & + \\
\hline 16 & 43.2 & $266 ; 284 \mathrm{sh} ; 346$ & Kaempferol derivative & + & & & + \\
\hline 17 & 49.8 & $256 ; 306 \mathrm{sh} ; 356 ; 370$ & Ellagic acid derivative & + & & & + \\
\hline PA & $23.5-47.0$ & $279-280$ & Proanthocyanidins & & + & + & \\
\hline
\end{tabular}

The peaks profile is shown in Fig. 2. The compositions of their three fractions eluted from a Sephadex LH-20 column $\left(F_{I}, F_{I I}\right.$ and $\left.F_{I I I}\right)$, as well as of the tannin-free decoction are also evidenced. +, compounds present in the fractions; sh, shoulder.

${ }^{a}$ Identification tentatively made by comparison of the retention times and/or UV spectra with authentic samples.

To confirm that correlation, the tannin-free decoction, that exhibited a very low DPPH scavenger activity as compared to the decoction (Fig. 1), was analysed by HPLC. Spectra corresponding to the proanthocyanidins were not detected, although other polyphenols were observed, as referred to Table 1, supporting evidence for a relationship between the decoction radical scavenger activity and its proanthocyanidins content.

\subsection{Antioxidant activity characterization}

\subsubsection{Interaction with superoxide anion}

The reactivity of decoction towards superoxide anion was evaluated by the decrease in the rate of nitroblue tetrazolium (NBT) reduction induced by $\mathrm{O}_{2}^{--}$generated by the enzymatic system xanthine oxidase/hypoxanthine. Any compound that decreases the rate of NBT reduction should react with $\mathrm{O}_{2}^{--}$unless itself reacts directly with NBT. The use of superoxide dismutase (SOD), the physiological scavenger of superoxide anion, enabled us to confirm the $\mathrm{O}_{2}^{--}$production in the assay, as it inhibited the reaction of NBT reduction in a concentration dependent manner (data not shown) with an inhibition concentration at $50 \%=3.47 \mathrm{U} \mathrm{ml}^{-1}$. Fig. 3 shows the results of the reactivities of different amounts of decoction against $\mathrm{O}_{2}^{--}$as expressed by SOD equivalent units with evidence for a linear correlation between decoction concentration and $\mathrm{O}_{2}^{--}$scavenging activity. Considering that the decoction neither reduced NBT by itself nor inhibited xanthine oxidase, as evaluated by uric acid formation (data not shown), the results denote the capacity of decoction for reacting with superoxide (Halliwell, 1997).

Although $\mathrm{O}_{2}^{--}$is a free radical, its reactivity is limited and it is enclosed in the compartment where it is produced. However, two molecules of superoxide anion rapidly dismutate to hydrogen peroxide and molecular

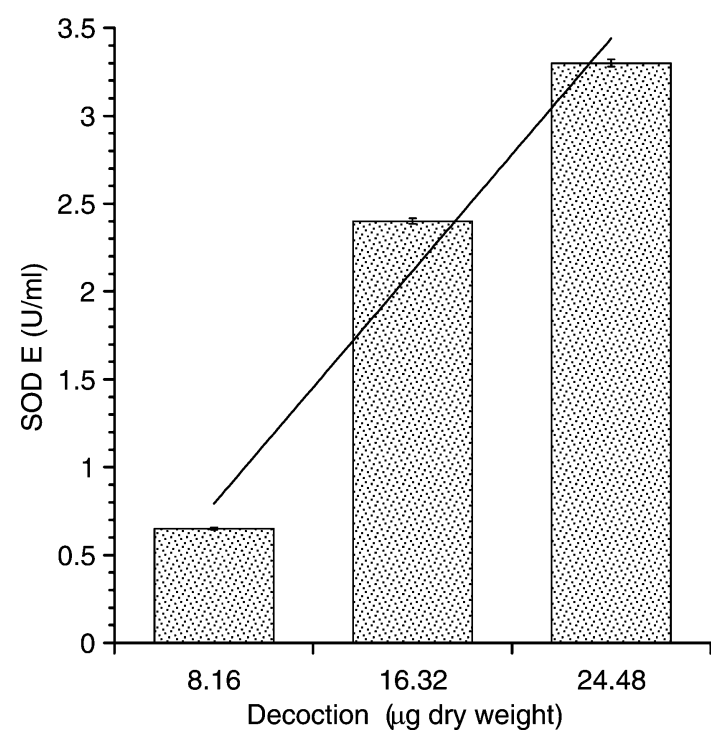

Fig. 3. Superoxide anion scavenging activity of Uncaria tomentosa decoction as evaluated by the inhibition of NBT reduction and expressed in terms of superoxide dismutase equivalent units (SOD E). Bars represent the mean $\pm \mathrm{SD}$ of at least three different experiments and the line evidences the linear correlation between superoxide anion scavenging capacity and the amount of decoction. 
Table 2

Scavenging of hydrogen peroxide, hydroxyl radical and hypochlorous acid by Uncaria tomentosa decoction

\begin{tabular}{llll}
\hline Decoction $(\mu \mathrm{g}$ dry weight) & \multicolumn{3}{c}{ Scavenger capacity $(\%)$} \\
\cline { 2 - 4 } & $\mathrm{H}_{2} \mathrm{O}_{2}$ & $\mathrm{OH}$ & $\mathrm{HOCl}$ \\
\hline 10.2 & & $32.4 \pm 3.5$ & \\
16.32 & $7.4 \pm 0.27$ & & $7.4 \pm 0.34$ \\
\hline
\end{tabular}

Data are expressed as the mean \pm standard deviation of at least three experiments. The scavenger capacity was expressed in percentage of decrease relatively to the control assay, i.e., the assay in the absence of added decoction.

oxygen, spontaneously or in a reaction catalysed by SOD. It can also react with nitric oxide (NO) to give peroxynitrite $\left(\mathrm{ONOO}^{-}\right)$, which is a highly cytotoxic species (Nordberg and Arnér, 2001).

\subsubsection{Interaction with hydrogen peroxide}

Hydrogen peroxide can be sensitively measured by the conventional peroxidase-based assay where guaiacol is used as an electron donor. The decoction reactivity with $\mathrm{H}_{2} \mathrm{O}_{2}$ was estimated by the decrease in absorbance at $436 \mathrm{~nm}$. In fact, any compound that reacts with $\mathrm{H}_{2} \mathrm{O}_{2}$ should decrease the rate of guaiacol oxidation decreasing the absorbance intensity at $436 \mathrm{~nm}$. In the presence of decoction $(16.32 \mu \mathrm{g}$ dry material) there was a slight decrease in absorbance at $436 \mathrm{~nm}$, denoting some interaction between decoction and $\mathrm{H}_{2} \mathrm{O}_{2}$, considering that decoction neither oxidized directly guaiacol nor was a substrate for peroxidase (data not shown). This decrease indicated a scavenger capacity of about $7 \%$, as shown in Table 2.

Although hydrogen peroxide is not a free radical it has a great physiological relevance because of its ability to penetrate biological membranes and to act like an intermediate in the production of more reactive oxygen species, namely hydroxyl radical and hypochlorous acid (Nordberg and Arnér, 2001).

\subsubsection{Interaction with hydroxyl radicals}

Hydroxyl radical is a highly reactive species attacking almost all biological molecules. It is formed from $\mathrm{H}_{2} \mathrm{O}_{2}$ in a reaction catalysed by metal ions $\left(\mathrm{Fe}^{2+}\right.$ or $\left.\mathrm{Cu}^{+}\right)$ known as the Fenton reaction (Nordberg and Arnér, 2001), where the superoxide anion plays an important role by reducing the metal ions.

$$
\mathrm{H}_{2} \mathrm{O}_{2}+\mathrm{Cu}^{+} / \mathrm{Fe}^{2+} \rightarrow \mathrm{HO}^{-}+\mathrm{OH}^{-}+\mathrm{Cu}^{2+} / \mathrm{Fe}^{3+}
$$

To test the interaction of the decoction with hydroxyl radicals, we used a simple assay where the radicals are formed in solution from the ascorbate/iron ion $/ \mathrm{H}_{2} \mathrm{O}_{2}$ system and are detected by their ability to degrade deoxyribose that, on heating with TBA, forms a pink chromogen (Payá et al., 1992). U. tomentosa decoction competed with deoxyribose for hydroxyl radicals demonstrating in vitro significant protective effect on the deoxyribose damage assay. Appropriate blanks were performed to rule out the possibility of decoction to affect the measurement of deoxyribose degradation (it had no effect when added to the TBA reagents), to enhance hydroxyl radical formation (it had no pro-oxidant effect) and to react with $\mathrm{HO}$ giving TBA-reactive substances (no chromogen formation in the absence of deoxyribose). Decoction showed a very good scavenging capacity towards HO* (Table 2) in comparison with mannitol (10.2 $\mu \mathrm{g}$ of decoction dry material presented a hydroxyl radical-scavenging capacity of about 30\% against about $40 \%$ for $1.8 \mathrm{mg}$ mannitol). Mannitol was used as a reference scavenger of hydroxyl radicals.

\subsubsection{Interaction with hypochlorous acid}

Hypochlorous acid $(\mathrm{HOCl})$ is produced in vivo, at inflammation sites by enzymatic oxidation of chloride ions. $\mathrm{HOCl}$ is much more toxic than either superoxide or hydrogen peroxide. It is a non-specific oxidising and chlorinating agent that reacts promptly with a variety of biological molecules (Winterbourn and Kettle, 2000). One of the major extracellular targets of $\mathrm{HOCl}$ attack is $\alpha_{1}$-antiproteinase, the main circulating inhibitor of serine proteases, as elastase. Thus, elastase activity may be used to evaluate either $\alpha_{1}$-antiproteinase activity or hypochlorous acid scavenging capacity. Actually, in the presence of $\mathrm{HOCl}$ the elastase activity is maximal, decreasing when previously we add a compound with hypochlorous acid scavenging capacity.

In the presence of decoction (16.32 $\mu \mathrm{g}$ dry material) the elastase activity was inhibited $7.4 \%$ (Table 2 ) denoting only a slight $\mathrm{HOCl}$-scavenging activity, especially, when compared with $N$-acetylcysteine. This reference compound, in our assay conditions, at the concentration of $54.8 \mu \mathrm{g}$, inhibited almost all the elastase activity $(99.6 \%)$. Control experiments showed that decoction neither inhibited elastase directly nor interfered with the capacity of $\alpha_{1}$-antiproteinase to inhibit elastase.

\subsubsection{Interaction with peroxyl radicals}

Peroxyl radicals are formed upon the reaction of carbon-centred radicals with oxygen and its formation is the major chain-propagation step in lipid peroxidation. They are biologically relevant active species with high capacity to damage cellular constituents (Cadenas, 1989).

Peroxyl radicals were generated in vitro by thermal decomposition of the hydrophilic azo initiator 2,2'-azobis-(2-amidinopropane hydrochloride) (AAPH) at a defined rate in aqueous solution leading to the immediate decay of the fluorescence intensity of B-phycoerythrin (Niki, 1990). However, in the presence of little amounts of decoction this fluorescence decay was decreased in a non-linear and concentration-dependent manner, as shown in Fig. 4. This non-linear fluorescence decay was similar to that of Trolox, a water soluble vitamin 


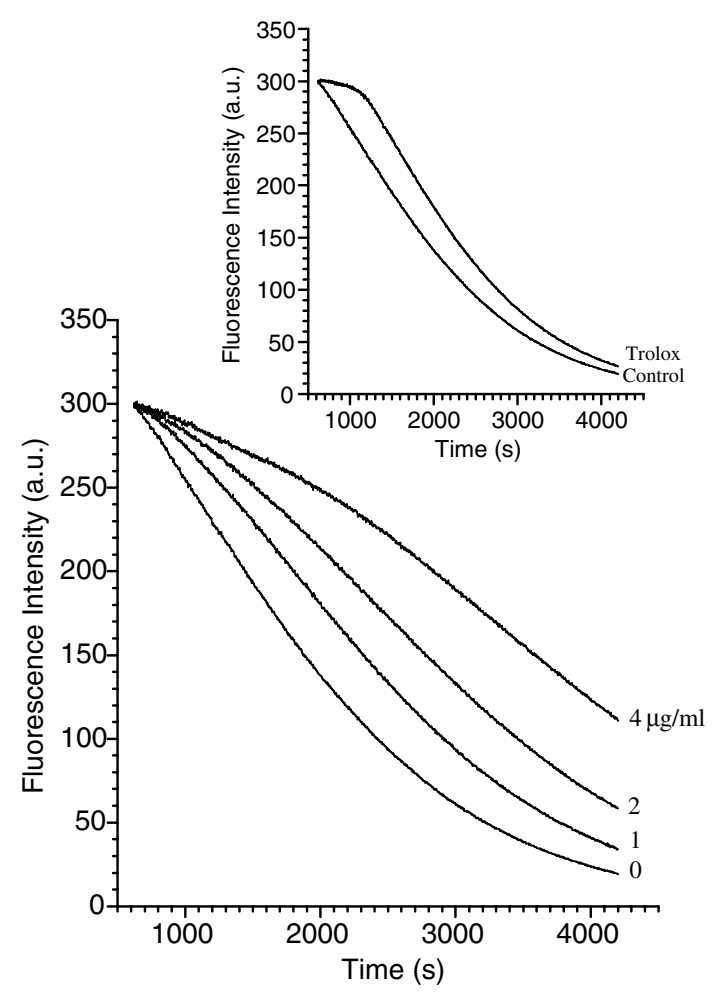

Fig. 4. Scavenging effect of Uncaria tomentosa decoction on AAPHinduced peroxyl radicals as assessed by the protection on the fluorescence decay of B-phycoerythrin. B-Phycoerythrin $(15 \mathrm{nM})$ in phosphate buffer $\mathrm{pH} 7.4$ was exposed to AAPH at $37^{\circ} \mathrm{C}$. Decoction, at different concentrations, was added immediately before AAPH as described in Section 4. The recordings are typical assays of at least three different experiments. Inset represents a typical assay where it is evident the effect of $3 \mu \mathrm{M}$ of Trolox $(0.8 \mu \mathrm{g} / \mathrm{ml})$.

E analogue and a well-known chain-breaking antioxidant (Niki, 1990). It caused an initial inhibition period of B-phycoerythrin fluorescence quenching, followed by a fluorescence decay similar to that of the control assay, as shown in the inset. Decoction did not induce a so clear initial inhibition period, but efficiently protected Bphycoerythrin from peroxyl radicals attack during the reaction time. Decoction is a mixture of constituents that probably react with ROO' in different ways, either as chain-breaking antioxidants, or as retardant antioxidants only decreasing the propagation rate like acetaminophen or salicylate (Dinis et al., 1994).

\subsubsection{Inhibition of lipid peroxidation}

The impact of decoction on lipid peroxidation was investigated on the basis of the effects on membrane lipid peroxidation induced by $\mathrm{Fe}^{2+} /$ ascorbate as evaluated by the production of thiobarbituric acid-reactive substances (TBARs). Lipid peroxidation in SR membranes, used as a model, increased with time, reaching an apparent maximum at 60 min (data not shown). Decoction significantly inhibited membrane lipid peroxidation, decreasing in about $86 \%$ the amount of TBARs formed
Table 3

SR membrane lipid oxidation promoted by $\mathrm{Fe}^{2+} /$ ascorbate $(20 \mu \mathrm{M} /$ $100 \mu \mathrm{M})$ in the absence and presence of decoction $(12.24 \mu \mathrm{g}$ dry weight), as measured by TBARs formation

\begin{tabular}{lll}
\hline $\begin{array}{l}\text { SR membranes in different } \\
\text { incubation systems }\end{array}$ & \begin{tabular}{l} 
TBARs \\
\cline { 2 - 2 }
\end{tabular} & $\begin{array}{l}\text { nmolMDA/mg } \\
\text { protein }\end{array}$ \\
\hline Native membranes & n.d. \\
$\mathrm{Fe}^{2+} /$ ascorbate & $15.52 \pm 0.32$ & \\
Decoction $+\mathrm{Fe}^{2+} /$ ascorbate & $2.18 \pm 0.014$ & 86 \\
\hline
\end{tabular}

Data are expressed as the mean \pm standard deviation of at least three experiments; n.d., not detected.

at 60 min relatively to the control assay, as indicated in Table 3. Although the measurement of thiobarbituric acid-reactive substances is a test lacking specificity, it is a very good indicative of SR lipid oxidation as previously demonstrated (Dinis et al., 1993, 1994; Soares et al., 1997).

The potential inhibitory effect of decoction on lipid peroxidation was evaluated by using iron as a metal ion catalyst. It has been suggested that the antioxidant activities of flavonoids depend on their reducing and chelating properties (Van Acker et al., 1996; Korkina and Afanas'ev, 1997). Therefore, the ability of decoction to inhibit lipid peroxidation may be exerted by complexing iron under a form catalytically silent. To clarify the antioxidant potential of $U$. tomentosa decoction in the presence of iron, the metal binding capacity of decoction was tested by assessing its absorption spectra in the absence and presence of iron. The results obtained (Fig. 5) show that the characteristic absorption peak of decoc-

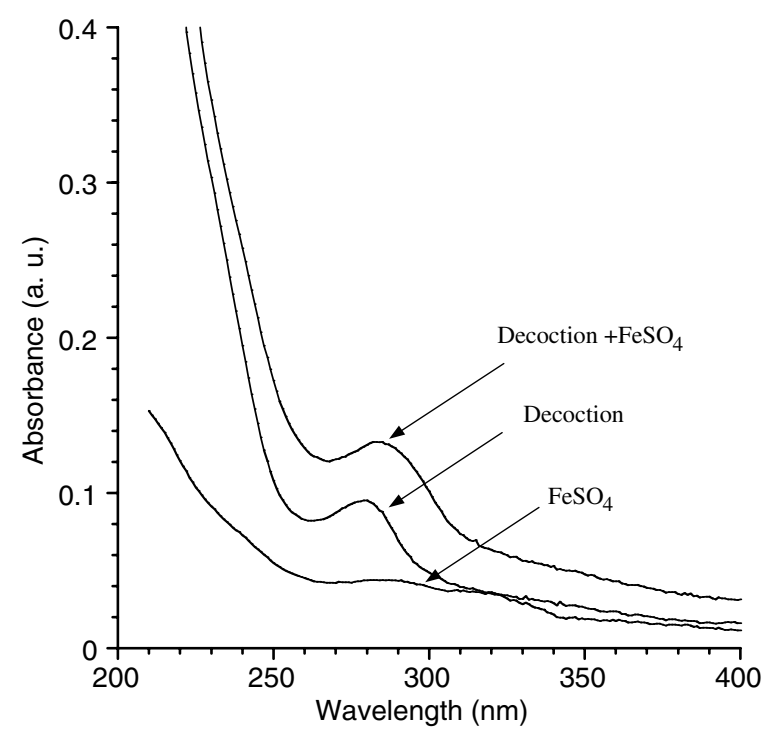

Fig. 5. The characteristic absorbance peak of Uncaria tomentosa decoction in the absence (decoction) and presence of iron (decoction $\left.+\mathrm{FeSO}_{4}\right)$. Ferrous sulphate $\left(\mathrm{FeSO}_{4}\right)$ alone does not present any peak at $280 \mathrm{~nm}$, the characteristic absorbance peak of decoction. 
tion at $280 \mathrm{~nm}$ was shifted to a higher wavelength, suggesting that decoction can bind iron under neutral $\mathrm{pH}$. Thus, the decoction antioxidant capacity, specifically the ability of HO' scavenging and the inhibition capacity of lipid peroxidation induced by the transition metal could be in part ascribed to a mechanism of iron binding, as described for the Ginseng extract (Zhang et al., 1996).

\section{Conclusion}

Despite cat's claw traditional use in various states of inflammation, the biochemical mechanisms underlying such properties remain unclear. Attempting to clarify some of those mechanisms we explored the capacity of reaction of a bark decoction against the oxidant species generated in the inflammatory process, as well as the involved chemical constituents.

Uncaria tomentosa bark decoction shows to be mainly composed by phenolic acids, essentially caffeic acid and proanthocyanidins, namely oligomeric procyanidins. The chemical structures of these phytochemicals include one or more aromatic rings bearing hydroxyl groups that are potentially able to act as reducing agents, as hydrogen donating antioxidants and as singlet oxygen quenchers (Rice-Evans et al., 1996). Owing to the basic chemical structure of its components, it would be expected that $U$. tomentosa bark decoction possessed a strong antioxidant activity. Actually, our data demonstrate such high antioxidant ability of the decoction, suggesting an important role of proanthocyanidins in its free radical scavenging properties, as clearly evidenced by the different effects of decoction and tannin-free decoction on the DPPH radical quenching (Fig. 1).

Furthermore, our data indicate that the decoction is a very good scavenger of superoxide anion and hydroxyl radical and in a lesser extension of hydrogen peroxide and hypochlorous acid. Additionally, it shows an efficient peroxyl scavenging activity when the radicals are formed in hydrophilic environment as well as a strong protective effect on membrane lipids against oxidation induced by the iron/ascorbate system. The decoction also shows some degree of metal-binding capacity which can explain in part its capacity for protecting membrane lipids from oxidation as well as its capacity of interaction with hydroxyl radicals. Noteworthy are the relatively very low amounts of decoction used in all the experiments to minimize the potential interference of decoction constituents with the reagents $(8-25 \mu \mathrm{g}$ decoction dry weight corresponding to $2.4-7.5 \mu \mathrm{g}$ proanthocyanidins).

In conclusion, our data provide a contribution for a better understanding of cat's claw anti-inflammatory activity and confirm some of the biological effects of its proanthocyanidins, namely the antioxidant and radical scavenging activities. Although preliminary studies about cytotoxicity have already been performed (Santa Maria et al., 1997), further research is required in order to make $U$. tomentosa decoction an effective anti-inflammatory agent.

\section{Experimental}

\subsection{Chemicals}

The azocompound 2,2'-azobis-(2-amidinopropane hydrochloride) (AAPH) was obtained from Polysciences, Inc. (Warrington, PA). 1,1-Diphenyl-2-picrylhydrazyl (DPPH), xanthine oxidase, hypoxanthine, nitroblue tetrazolium, superoxide dismutase (SOD), guaiacol, horseradish peroxidase, deoxyribose, elastase, $\alpha_{1}$-antiproteinase, elastase substrate, $N$-acetylcysteine and B-phycoerythrin were purchased from Sigma-Aldrich Química S.A. (Portugal). Ascorbic acid was obtained from Fluka (Switzerland). The reference compounds, caffeic acid (minimum 99\%), (+)-catechin hydrate (minimum 98\%), $p$-coumaric acid, ellagic acid $(\approx 95 \%)$, (-)-epicatechin, kaempferol (minimum 90\%), protocatechuic acid, quercetin dihydrate (minimum 98\%), syringic acid (minimum 98\%) and vanillic acid were purchased from Sigma-Aldrich. The cyanidin chloride was obtained from Extrasynthese. All the other chemicals were of grade for biochemical analysis. Deionized water was used throughout and when necessary Ultrapure Milli Q water was also used.

Sarcoplasmic reticulum vesicles were prepared from rabbit white muscles as previously described (Dinis et al., 1993).

\subsection{Plant material}

Uncaria tomentosa barks (Willd) DC. were kindly supplied by $\mathrm{Dr}^{\mathrm{a}}$ Olga Lock De Ugaz, from Pontificia Universidad Católica, Lima, Peru.

Voucher specimen is deposited at the Laboratory of Pharmacognosy, Faculty of Pharmacy, University of Coimbra.

\subsection{Preparation of Uncaria extracts}

Decoction was prepared according to the traditional Peruvian medicine, by boiling $0.750 \mathrm{~g}$ of powdered bark in distilled water $(150 \mathrm{ml})$ for $40 \mathrm{~min}$. After cooling, the final volume was adjusted to $250 \mathrm{ml}$ and the marc was eliminated by filtration.

A tannin-free decoction was also prepared from aliquots of decoction $(10 \mathrm{ml})$ treated with hide powder $(100 \mathrm{mg})$, according to the guidelines for the tannins determination in Ratanhiae radix (Council of Europe, 
2002). After centrifugation, the resulting solution was filtered and the free radical scavenging activity and HPLC phenolic profile were evaluated.

In order to establish the phenolic profile of $U$. tomentosa bark decoction and its in vitro antioxidant and radical scavenging activity, four decoctions were prepared as described above and mixed together. Seven hundred millilitres were taken and the lipids were eliminated with $n$-hexane (1.5:1, v/v, three times). The aqueous phase was concentrated under reduced pressure almost to dryness and dissolved in methanol-water $(2: 3, \mathrm{v} / \mathrm{v})$ to a final volume of $5 \mathrm{ml}$. A standardized extract, containing $30 \mathrm{mg}$ of proanthocyanidins, expressed as $\mathrm{mg}$ catechin equivalent by $100 \mathrm{mg}$ of decoction dry weight, was obtained. This extract is referred as decoction along the work.

\subsection{Determination of proanthocyanidins}

Proanthocyanidins contents were determined by spectroscopy at $500 \mathrm{~nm}$, on the basis of a colorimetric reaction with vanillin- $\mathrm{HCl}$, as described by JulkunenTitto (1985). The results were expressed as $\mathrm{mg}$ of catechin equivalent/g of dry plant material.

\subsection{Gel chromatography fractionation}

An aliquot of the decoction $(4 \mathrm{ml})$ was fractionated by gel chromatography on a Sephadex LH-20 column $(25-100 \mu \mathrm{m}, 170 \times 20 \mathrm{~mm})$, from Sigma-Aldrich. The elution was performed using $\mathrm{MeOH}-\mathrm{H}_{2} \mathrm{O}(1: 1) 150 \mathrm{ml}$, $\mathrm{MeOH}-\mathrm{H}_{2} \mathrm{O}$ (7:3) $150 \mathrm{ml}$ and acetone- $\mathrm{H}_{2} \mathrm{O}$ (7:3) $100 \mathrm{ml}$. Nine fractions were obtained according to their behaviour under UV observation. All fractionas were concentrated under vacuum and dissolved in $\mathrm{MeOH}_{-}$ $\mathrm{H}_{2} \mathrm{O}(1: 1)$ to a final volume of $1 \mathrm{ml}$.

\subsection{Analytical HPLC}

HPLC analyses were carried out in a Gilson instrument equipped with a photodiode-array detector (DAD) and an ODS-2 column $(5 \mu \mathrm{m}$, $250 \mathrm{~mm} \times 4.6 \mathrm{~mm}$ i.d.) from Waters. A mobile phase constituted by methanol (A) and 5\% aqueous formic acid, v/v (B) was used. The gradient consisted of 5$15 \% \mathrm{~A}$, for $10 \mathrm{~min}, 15-35 \% \mathrm{~A}$ for more $25 \mathrm{~min}$, then from $35 \%$ to $50 \% \mathrm{~A}$, for $15 \mathrm{~min}$ and finally $50-100 \%$ A for $10 \mathrm{~min}$ in a total of $60 \mathrm{~min}$, at a flow-rate of $1 \mathrm{ml} \mathrm{min}-1$. Chromatographic profiles were recorded at 280 and $320 \mathrm{~nm}$. Data treatment was carried out with a software Unipoint ${ }^{\mathrm{R}}$.

The compounds were identified by their retention times and UV spectra, comparing them with authentic samples. For some compounds, their confirmation was performed by the addition of authentic samples to aliquots of the decoction or tannin-free decoction.

\subsection{Thin layer chromatography}

TLC was carried out as described by Escribano-Bailón et al. (1992) to classify the proanthocyanidins according to their polymerisation degree. Pre-coated silica gel $60 \mathrm{~F}_{254}$ plates from Merck and toluene-acetoneacetic acid (3:7.5:1) were used as stationary and mobile phases, respectively. Detection was made with a solution of $1 \%$ vanillin in $70 \%$ hydrochloric acid. Catechin was used as a reference compound.

\subsection{In vitro antioxidant and radical scavenging activity}

\subsubsection{Measurement of free radical scavenger activity}

Free radical scavenging activities of decoction and tannin-free decoction (reported to $280 \mu \mathrm{g}$ of plant dry weight) were evaluated by their reactivities with an ethanolic solution of $500 \mu \mathrm{M}$ DPPH from the change in absorbance at $517 \mathrm{~nm}$, according to the method of Blois (1958). The results were expressed in percentage of DPPH reduction, as evaluated by the decrease in the absorbance value at $517 \mathrm{~nm}$ as compared to the control assay, i.e., the assay without the sample under study.

\subsubsection{Superoxide anion scavenging activity}

Superoxide anions were generated enzymatically (Payá et al., 1992) at room temperature by adding $0.042 \mathrm{U} \mathrm{ml}^{-1}$ of xanthine oxidase to a mixture of $2 \mathrm{ml}$

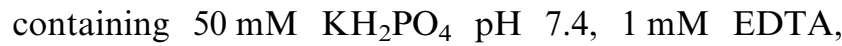
$100 \mu \mathrm{M}$ hypoxanthine and $100 \mu \mathrm{M}$ NBT. The rate of NBT reduction was recorded during $150 \mathrm{~s}$ in a PerkinElmer Lambda 6 Spectrophotometer, at $560 \mathrm{~nm}$. The decoction was added at several concentrations and the results were expressed as SOD equivalent units.

Control experiments were performed to determine whether the decoction directly reduced NBT or inhibited xanthine oxidase, as described by Soares et al. (1997).

Superoxide dismutase was used as a reference scavenger of superoxide anion.

\subsubsection{Hydrogen peroxide scavenging activity}

Hydrogen peroxide was measured by the formation of a brown colour, recorded at $436 \mathrm{~nm}$, in a reaction mixture of $1 \mathrm{ml}$ containing $150 \mathrm{mM} \mathrm{KH}_{2} \mathrm{PO}_{4}-\mathrm{KOH}$ buffer $\mathrm{pH}$ 7.4, $100 \mu \mathrm{l}$ guaiacol solution (obtained by adding $100 \mu \mathrm{l}$ of pure guaiacol to $50 \mathrm{ml}$ desionized water) and $5 \mu \mathrm{l}$ horseradish peroxidase $(5 \mathrm{mg} / \mathrm{ml}$ in the same phosphate buffer) as described elsewere (Aruoma et al., 1989). The reaction was started by $\mathrm{H}_{2} \mathrm{O}_{2}$ addition. Decoction was pre-incubated with $\mathrm{H}_{2} \mathrm{O}_{2}$ for $30 \mathrm{~min}$ at $25^{\circ} \mathrm{C}$ and then, aliquots were removed and assayed for the remaining $\mathrm{H}_{2} \mathrm{O}_{2}$. Adequate blanks were prepared to determine whether the sample vehicle interfered with the reaction or if decoction was a substrate for peroxidase. 


\subsubsection{Hydroxyl radicals scavenging activity}

Hydroxyl radicals were generated by the ascorbate/ iron $/ \mathrm{H}_{2} \mathrm{O}_{2}$ system, essentially as referred elsewhere (Halliwell et al., 1987; Burits and Bucar, 2000). The reaction mixture in a final volume of $1 \mathrm{ml}$, contained $20 \mathrm{mM} \mathrm{KH}{ }_{2} \mathrm{PO}_{4}-\mathrm{KOH}$ buffer pH 7.4, $2.8 \mathrm{mM}$ 2-deoxyribose, $\mathrm{FeCl}_{3}$-EDTA (100 and $104 \mu \mathrm{M}$, respectively), $1 \mathrm{mM} \mathrm{H}_{2} \mathrm{O}_{2}$ and $100 \mu \mathrm{M}$ ascorbate. After an incubation period of $1 \mathrm{~h}$ at $37^{\circ} \mathrm{C}$ the extent of deoxyribose degradation was measured by the thiobarbituric acid method (Buege and Aust, 1978). Decoction, vehicle (blank) or D-manitol (reference compound) were added before ascorbate addition. Solutions of iron salt, $\mathrm{H}_{2} \mathrm{O}_{2}$ and ascorbate were always prepared just before use.

\subsubsection{Hypochlorous acid scavenging activity}

Hypochlorous acid $(\mathrm{HOCl})$ was prepared immediately before use and the $\mathrm{HOCl}$ scavenging activity was indirectly evaluated by the measurement of elastase activity, as described by Payá et al. (1992). Control experiments were also carried out to eliminate any direct effect of decoction on $\alpha_{1}$-antiproteinase or elastase activities. $N$-Acetylcysteine was used as a reference scavenger of $\mathrm{HOCl}$ (Haenen and Bast, 1991). Briefly, decoction was mixed with $\alpha_{1}$-antiproteinase $(0.77 \mathrm{mg})$ in phosphate buffer and $\mathrm{HOCl}$ was added at a final concentration of $75 \mu \mathrm{M}$. This reaction mixture was incubated at $25^{\circ} \mathrm{C}$ for $1 \mathrm{~h}$ and then elastase was added. After incubation for further $30 \mathrm{~min}$, the remaining elastase activity was evaluated by the change in absorbance at $410 \mathrm{~nm}$, using $N$-succinyl-ala-ala-ala- $p$-nitroanilide as substrate.

\subsubsection{Peroxyl radicals scavenger activity}

To assess the capacity of decoction as a scavenger of peroxyl radicals generated in aqueous phase, we used the water-soluble azo initiator AAPH. AAPH generates peroxyl radicals at a constant rate by thermal decomposition, causing a decrease of B-phycoerythrin fluorescence intensity (Maitra et al., 1995). B-Phycoerythrin $(15 \mathrm{nM})$ was incubated at $37^{\circ} \mathrm{C}$ in $2 \mathrm{ml}$ of $0.2 \mathrm{M}$ phosphate buffer $\mathrm{pH} 7.4$ with continuous stirring. Decoction or Trolox (a reference compound) were added $30 \mathrm{~s}$ before addition of $10 \mathrm{mM}$ AAPH and the peroxyl scavenger activity was evaluated by the quenching of B-phycoerythrin fluorescence as previously described (Maitra et al., 1995). The fluorescence measurements were carried out at $37^{\circ} \mathrm{C}$ during $1 \mathrm{~h}$ in a Perkin-Elmer computer controlled LS 50 spectrofluorimeter. The excitation and emission wavelengths were 540 and $575 \mathrm{~nm}$, respectively (bandpasses $5 \mathrm{~nm}$ ).

\subsubsection{Antioxidant activity against membrane lipid peroxidation}

Sarcoplasmic reticulum membranes were used, as a native membrane model, to assess the effect of decoction on lipid peroxidation, as previously described (Dinis et al., 1994). Briefly, SR membranes ( $2 \mathrm{mg}$ protein $/ \mathrm{ml}$ ) in a medium containing $10 \mathrm{mM}$ TRIS, $150 \mathrm{mM} \mathrm{NaCl}$, $\mathrm{pH}$ 7.0, were incubated under magnetic stirring at $37^{\circ} \mathrm{C}$, for $90 \mathrm{~min}$, in the presence of $20 \mu \mathrm{M} \mathrm{FeSO} 4$ and $100 \mu \mathrm{M}$ ascorbate. Decoction or vehicle was added before the peroxidation inducers. The extent of oxidation and the decoction antioxidant potential were evaluated by the production of TBARs (Buege and Aust, 1978). Butylated hydroxytoluene was added to the TBA reagent to prevent additional chromophore formation during the assay procedure.

\subsubsection{Capacity of binding $\mathrm{Fe}^{2+}$}

The binding of iron ions by the decoction was investigated spectrophotometrically. Spectra of the decoction before and after addition of $80 \mu \mathrm{M} \mathrm{FeSO}_{4}$ in TRIS buffer $\mathrm{pH} 7.0$ were recorded at room temperature in a Perkin-Elmer Lambda 6 spectrophotometer, between 210 and $400 \mathrm{~nm}$ and compared with that of $\mathrm{FeSO}_{4}$ recorded in the same conditions.

\section{Acknowledgements}

This research was funded in part by the Fundação para a Ciência e Tecnologia, Programa Operacional de Ciência, Tecnologia e Inovação (POCTI) and POCTI/ 35019/AGR/2000. The authors also thank Prof. Leonor Almeida for her advice and many helpful suggestions.

\section{References}

Aquino, R., De Feo V, Simone, F., Pizza, C., Cirino, G., 1991. Plant metabolites. New compounds and anti-inflammatory activity of Uncaria tomentosa. J. Nat. Prod. 54, 453-459.

Arnao, M.B., Cano, A., Acosta, M., 2001. The hydrophilic and lipophilic contribution to total antioxidant activity. Food Chem. $73,239-244$.

Aruoma, O.I., Halliwell, B., Hoey, B.M., Butler, J., 1989. The antioxidant action of $\mathrm{N}$-acetylcysteine: its reaction with hydrogen peroxide, hydroxyl radical, superoxide and hypochlorous acid. Free Radic. Biol. Med. 6, 593-597.

Bagchi, D., Bagchi, M., Stohs, S.J., Das, D.K., Ray, S.D., Kuszynski, C.A., Joshi, S.S., Pruess, H.G., 2000. Free radicals and grape seed proanthocyanidin extract: importance in human health and disease prevention. Toxicology 148, 187-197.

Blois, M.S., 1958. Antioxidant determinations by the use of a stable free radical. Nature 181, 1199-1200.

Bonina, F., Puglia, C., Tomaino, A., Saija, A., Mulinacci, N., Romani, A., Vincieri, F.F., 2000. In-vitro antioxidant and in-vivo photoprotective effect of three lyophilized extracts of Sedum telephium L. leaves. J. Pharm. Pharmacol. 52, 1279-1285.

Buege, J.A., Aust, S.D., 1978. Microssomal lipid peroxidation. Methods Enzymol. 52, 302-310.

Burits, M., Bucar, F., 2000. Antioxidant activity of Nigella sativa essential oil. Phytother. Res. 14, 323-328.

Cadenas, E., 1989. Biochemistry of oxygen toxicity. Annu. Rev. Biochem. 58, 79-110. 
Conner, E.M., Grisham, M.B., 1996. Inflammation, free radicals and antioxidants. Nutrition 12, 274-277.

Council of Europe, 2002. European Pharmacopoeia. Council of Europe, Strasbourg, pp. 1857-1858.

Desmarchelier, C., Mongelli, E., Coussio, J., Ciccia, G., 1997. Evaluation of the in vitro antioxidant activity in extracts of Uncaria tomentosa (Willd.) DC. Phytother. Res. 11, 254-256.

Dinis, T.C.P., Almeida, L.M., Madeira, V.M.C., 1993. Lipid peroxidation in sarcoplasmic reticulum membranes: effect on functional and biophysical properties. Arch. Biochem. Biophys. 301, 256-264.

Dinis, T.C.P., Madeira, V.M.C., Almeida, L.M., 1994. Action of phenolic derivatives (acetaminophen, salicylate, and 5-aminosalicylate) as inhibitors of membrane lipid peroxidation and as peroxyl radical scavengers. Arch. Biochem. Biophys. 315, 161-169.

Escribano-Bailón, T., Gutiérrez-Fernández, Y., Rivas-Gonzalo, J.C., Santos-Buelga, C., 1992. Characterization of procyanidins of Vitis vinifera variety Tinta del País grape seeds. J. Agric. Food Chem. 40, 1794-1799.

Groot, H., Rauen, U., 1998. Tissue injury by reactive oxygen species and the protective effects of flavonoids. Fundam. Clin. Pharmacol. 12, 249-255.

Haenen, G.R.M.M., Bast, A., 1991. Scavenging of hypochlorous acid by lipoic acid. Biochem. Pharmacol. 42, 2244-2246.

Halliwell, B., 1997. Antioxidants: The basics — what they are and how to evaluate them. In: Sies, H. (Ed.), Advances in Pharmacology. Academic Press, London, pp. 3-20.

Halliwell, B., Gutteridge, J.M.C., Aruoma, O.I., 1987. The deoxyribose method: a simple "test-tube" assay for determination of rate constants for reactions of hydroxyl radicals. Anal. Biochem. 165, 215-219.

Halliwell, B., Hoult, J.R., Blake, D.R., 1988. Oxidants, inflammation and anti-inflammatory drugs. FASEB J. 2, 2867-2873.

Julkunen-Titto, R., 1985. Phenolic constituents in the leaves of northern willows: methods for the analysis of certain phenolics. J. Agric. Food Chem. 33, 213-217.

Korkina, L.G., Afanas'ev, I.B., 1997. Antioxidant and chelating properties of flavonoids. In: Sies, H. (Ed.), Advances in Pharmacology. Academic Press, London, pp. 151-163.

Laus, G., Keplinger, D., 1994. Separation of stereoisomeric oxindole alkaloids from Uncaria tomentosa by high-performance liquid chromatography. J. Chromatogr. A 662, 243-249.

Li, W.G., Zhang, X.Y., Wu, Y.J., Tian, X., 2001. Anti-inflammatory effect and mechanism of proanthocyanidins from grape seeds. Acta Pharmacol. Sin. 22, 1117-1120.

Maitra, I., Marcocci, L., Droy-Lefaix, M.T., Packer, L., 1995. Peroxyl radical scavenging activity of Ginkgo biloba extract Egb 761. Biochem. Pharmacol. 49, 1649-1655.

Maxwell, S.R.J., 1995. Prospects for the use of antioxidant therapies. Drugs 49, 345-361.

Mohamed, A-F., Matsumoto, K., Tabata, K., Takayama, H., Kitajima, M., Aimi, N., Watanabe, H., 2000. Effects of Uncaria tomentosa total alkaloid and its component on experimental amnesia in mice: elucidation using the passive avoidance test. J. Pharm. Pharmacol. 52, 1553-1561.

Muhammad, I., Dunbar, D.C., Khan, R.A., Ganzera, M., Khan, I.A., 2001. Investigation of Uña De Gato I. 7-Deoxyloganic acid and
${ }^{15} \mathrm{~N}$ NMR spectroscopic studies on pentacyclic oxindole alkaloids from Uncaria tomentosa. Phytochemistry 57, 781-785.

Munasinghe, T.C.J., Seneviratne, C.K., Thabrew, M.I., Abeysekera, A.M., 2001. Antiradical and antilipoperoxidative effects of some plant extracts used by Sri Lankan traditional medical practitioners for cardioprotection. Phytother. Res. 15, 519-523.

Niki, E., 1990. Free radical initiators as source of water- or lipidsoluble peroxyl radicals. Methods Enzymol. 186, 100-108.

Nordberg, J., Arnér, E.S.J., 2001. Reactive oxygen species, antioxidants, and the mammalian thioredoxin system. Free Radic. Biol. Med. 31, 1287-1312.

Packer, L., Rimbach, G., Virgili, F., 1999. Antioxidant activity and biologic properties of a procyanidin-rich extract from pine (Pinus maritima) bark, pycnogenol. Free Radic. Biol. Med. 27, 704-724.

Payá, M., Halliwell, B., Hoult, J.R.S., 1992. Interactions of a series of coumarins with reactive oxygen species. Biochem. Pharmacol. 44, 205-214.

Piscoya, J., Rodriguez, Z., Bustamante, S.A., Okuhama, N.N., Miller, M.J., Sandoval, M., 2001. Efficacy and safety of freeze-dried cat's claw in osteoarthritis of the knee: mechanisms of action of the species Uncaria guianensis. Inflamm. Res. 50, 442-448.

Rice-Evans, C.A., Miller, N.J., Paganga, G., 1996. Structure-antioxidant activity relationships of flavonoids and phenolic acids. Free Radic. Biol. Med. 20, 933-956.

Rizzi, R., Re, F., Bianchi, A., De Feo, V., Simone, F., Bianchi, L., Stivala, L.A., 1993. Mutagenic and antimutagenic activities of Uncaria tomentosa and its extracts. J. Ethnopharmacol. 38, 63-77.

Sandoval, M., Charbonnet, R.M., Okuhama, N.N., Roberts, J., Krenova, Z., Trentacosti, A.M., Miller, M.J.S., 2000. Cat's claw inhibits $\mathrm{TNF} \alpha$ production and scavenges free radicals: role in cytoprotection. Free Radic. Biol. Med. 29, 71-78.

Santa Maria, A., Lopez, A., Diaz, M.M., Alban, J., Galan de Mera, A., Orellana, J.A., Pozuelo, J.M., 1997. Evaluation of the toxicity of Uncaria tomentosa by bioassays in vitro. J. Ethnopharmacol. 57, 183-187.

Santos-Buelga, C., Scalbert, A., 2000. Proanthocyanidins and tanninlike compounds-nature, occurrence, dietary intake and effects on nutrition and health. J. Sci. Food Agric. 80, 1094-1117.

Sheng, Y., Pero, R.W., Amiri, A., Bryngelsson, C., 1998. Induction of apoptosis and inhibition of proliferation in human tumor cells treated with extracts of Uncaria tomentosa. Anticancer Res. 18, 3363-3368.

Soares, J.R., Dinis, T.C.P., Cunha, A.P., Almeida, L.M., 1997. Antioxidant activities of some extracts of Thymus zygis. Free Radic. Res. 26, 469-478.

Van Acker, S.A.B.E., Van Den Berg, D., Tromp, M.N.J.L., Griffioen, D.H., Van Bennekom, W.P., Van Der Vijgh, W.J.F., Bast, A., 1996. Structural aspects of antioxidant activity of flavonoids. Free Radic. Biol. Med. 20, 331-342.

Winterbourn, C.C., Kettle, A.J., 2000. Biomarkers of myeloperoxidase-derived hypochlorous acid. Free Radic. Biol. Med. 29, 403409.

Zhang, D., Yasuda, T., Yu, Y., Zheng, P., Kawabata, T., Ma, Y., Okada, S., 1996. Ginseng extract scavenges hydroxyl radical and protects unsaturated fatty acids from decomposition caused by iron-mediated lipid peroxidation. Free Radic. Biol. Med. 20, 145150. 